\title{
Dix ans de Politique européenne de sécurité et de défense
}

\author{
La gestion des conflits au service du non-choix \\ stratégique?
}

\begin{abstract}
Galia Glume

Keywords: Crisis management, Peacekeeping, European security strategy, CFSP/ESDP (CSDP), European interest.

Ten years from 1999 Helsinki Headline Goal, the European security and defence policy (ESDP) provided the European Union with an in-depth policy for international crisis management and peacekeeping. By developing common capabilities to intervene into crises and conflicts in Europe and beyond, the EU Member States made the choice of autonomy - despite the stricto sensu Defence of Europe has no doubt remained NATO prerogative. Both civil and military, ESDP instruments nevertheless allow a comprehensive action to back the EU diplomacy - as EU peace-support missions since 2003 do suggest: in the Balkans or DR Congo, Aceh Indonesia or in Georgia, Caucasus. Whither ESDP, the European security strategy relies on further leverage: economical, financial, political or normative policies shape a wider EU external action. However, can ESDP be qualified as an instrument for a European foreign policy? Beyond a consensus a minima on the importance of peace, could it entail an instrument that could serve a European interest: a common interest that would need more than a 'conflict management-dedicated' ESDP?
\end{abstract}

Mots-clés : Gestion des crises, Maintien de la paix, Stratégie européenne de sécurité, PESC/PESD (PSDC), Intérêt européen.

Dix ans après son lancement à Helsinki, la politique européenne de sécurité et de défense (PESD) a doté l'Union européenne d'une véritable politique de gestion de crises et de maintien de la paix. En développant des capacités communes pour intervenir dans les crises et les conflits en Europe et au-delà, les vingt-sept ont choisi l'autonomie, même si la défense de l'Europe reste la prérogative de l'Organisation du traité de l'atlantique nord. À la fois civils et militaires, les instruments de la PESD permettent une action large, qui soutient la diplomatie de l'Union - comme le suggèrent les nombreuses missions menées depuis 2003 dans les Balkans, en République démocratique du Congo, à Aceh ou dans le Caucase. Au-delà de la PESD, la Stratégie européenne de sécurité s'appuie également sur des leviers d'influence économiques, financiers, politiques, 
normatifs... au service de l'action externe de l'Union. Mais la PESD constitue-t-elle vraiment un outil de politique étrangère? Un instrument au service d'un intérêt proprement européen : un intérêt commun qui déborderait le cadre et les objectifs de la gestion des conflits?

\section{Introduction}

Lors sa naissance il y a dix ans, le principal débat qui animait la scène européenne quant au sort de la Politique européenne de sécurité et de défense (PESD), c'était certainement de savoir si celle-ci allait déboucher sur une « défense européenne », ou si elle se cantonnerait aux missions de maintien de la paix en Europe. En partant de l'accord de 1998-1999 sur la nécessité, pour les États-membres de l’UE, de se doter d'une capacité militaire commune et autonome pour répondre aux crises touchant l'Europe, ne pouvait-on pas escompter un spill over, c'est-à-dire un débordement de la coopération, du domaine du maintien de la paix au domaine de la défense à proprement parler ? Il était évident alors pour de nombreux observateurs de cet «ovni» qu'était la jeune PESD, qu'elle était tout sauf le synonyme d'une capacité de défense européenne en marche. À l'instar d'États-membres comme la France ou la Belgique, certains analystes souhaitaient pourtant y voir une brèche dans le domaine réservé de l'OTAN, un futur levier de politique étrangère.

Dix ans après son lancement à Helsinki, on en est encore loin. Ce projet, qui a pourtant connu certaines avancées rapides et substantielles, demeure loin d'être voulu par tous, et l'«Europe puissance » n'est pas à l'agenda. D'autres déboires de la construction européenne - dont les questions militaires ou de sécurité ont d'ailleurs fait l'objet pendant près de quatre décennies - comme le rejet du traité constitutionnel par la France et les Pays-Bas, ont même, ces dernières années, éclipsé la problématique au profit de questionnements plus existentiels. Le traité de Lisbonne, par lequel la PESD change de nom, pour devenir la PSDC - la Politique européenne de sécurité et de défense devient la Politique de sécurité et de défense commune - entérine quelques avancées mais n'est pas synonyme de révolution en la matière ${ }^{1}$.

\footnotetext{
${ }^{1}$ Ce changement de terminologie intervient fin 2009, à l'occasion de l'entrée en vigueur du Traité. Cependant, par souci de clarté, nous n'employons que le terme «PESD» au long de cet exposé, qui constitue une tentative de bilan critique sur dix ans d'une réalité nouvelle, la PESD, incluant quelques mois sous sa nouvelle appellation, la PSDC. À cet égard, nous nous permettons de remercier les autorités européennes du moment, qui n’ont pas hésité une fois encore à compliquer le parcours des citoyens - et des étudiants notamment - pour appréhender l'ingénierie institutionnelle et le subtil jargon de la construction européenne.
} 
Cela ne signifie pas que l’Union reste dépourvue de moyens militaires autonomes ; en outre, sa capacité opérationnelle est désormais également civile. À ce jour, les Européens ne sont plus seulement la multitude chaotique ou l'Europe «impuissante » tant galvaudée des années Bosnie. De nombreuses opérations militaires et civiles ont été lancées, dans le cadre d'une politique qui couvre désormais l'entièreté du spectre des conflits et des crises : prévention, gestion et reconstruction. Une politique dont les discours et le concept de sécurité de l'UE forcent la cohérence, et dont la PESD ne représente que l'un des instruments les plus visibles.

A la question : " une PESD, pour quoi faire ? », les Européens semblent en effet avoir affiné leur réponse. Aujourd'hui, il est clair que la défense de l'Europe reste la prérogative de l'Alliance atlantique et que les missions de la PESD se focalisent presqu'uniquement sur le maintien de la paix et la gestion multinationale des crises. Dès lors, le débat semble se déplacer : la PESD sert-elle uniquement à l'UE de cadre pour une politique globale de gestion des conflits, en soutien à l'ONU notamment, ou peutelle être mise au service d'objectifs de politique étrangère - d'une politique étrangère $e u$ ropéenne?

Les soldats et gendarmes qui partent patrouiller le long des lignes de cessez-le-feu du Caucase ou rassurent une "paix sans réconciliation» dans les Balkans, les fonctionnaires qui œuvrent au Kosovo ou entraînent les policiers afghans : Servent-ils le consensus mou d'une «Europe plus sûre dans un monde meilleur ${ }^{2}$ » ou revêtent-ils aussi les traits d'un outil stratégique, aidant à convaincre les partenaires de l'Union, soutenant ses choix diplomatiques communs, cimentant les failles d'une puissance trop économique et pas assez politique ? En ce qui concerne la gestion des conflits, les États-membres de l'UE sont bel et bien sur la voie de l'action - quelle que soit la portée de cette action. Il n'est pas sûr cependant que la PESD, telle qu'elle s'est développée depuis une décennie, leur offre un instrument décisif au service d'une politique étrangère qui, elle aussi, demeure le plus souvent au stade de processus.

\section{Back to Basics : les origines}

À travers le lancement d'une vingtaine de missions, l'Union européenne s'est taillée une part de choix en tant qu'acteur du maintien de la paix dans le monde, parmi les organisations régionales qui en ont fait l'une de leurs prérogatives. À côté de l'OTAN, d'abord, qui dans le même temps a délaissé son rôle de substitut aux casques bleus dans les Balkans pour se consacrer à des opérations extérieures bien plus proches de la

\footnotetext{
2 Titre de la Stratégie européenne de sécurité, Bruxelles, décembre 2003. Notons que la Stratégie
} européenne de sécurité a fait l'objet d'un bilan lié à sa mise en œuvre en décembre 2008. 
guerre - sous le nouveau vocable «opérations de stabilisation» (Durch \& Berkman 2006 : 43). À côté de l'OSCE aussi, qui assume des mandats civils de gestion de crises en Eurasie. À côté, enfin, de l'Union africaine, qui parallèlement aux européens a commencé à se doter de forces de paix régionales, certes moins bien loties en termes d'entraînement, de capacités ou de financement. Ces organisations ont toutes suivi le mouvement lancé par l'OTAN dans les années 1990, lorsque les opérations de maintien de la paix de l'ONU sont entrées dans une crise sans précédent, à la lumière crue du génocide au Rwanda ou du massacre de Srebrenica : la régionalisation du maintien de la paix.

Les européens, lorsqu'ils donnent une consistance à la Politique européenne de sécurité et de défense, en 1999, s'inscrivent donc dans une série d'évolutions à l'œuvre au sein du système de sécurité collective global des Nations unies. Toutefois, pour l'Union européenne, si la PESD doit lui permettre de s'engager sur certains théâtres, entre autres militairement, sans dépendre de l'OTAN ou de l'ONU, elle doit aussi constituer un moyen de soutenir sa diplomatie propre : Être capable, de façon autonome, d'intervenir selon ses propres intérêts de sécurité.

Au-delà d'une certaine européanisation des questions de sécurité, il ne s'agit cependant pas de faire concurrence à l'OTAN. Certes, alors que l'Union de l'Europe occidentale $(\mathrm{UEO})^{3}$ est dissoute au profit de la PESD, son article 5 - établissant la clause de défense collective au sein de ses États-membres - ne tombera pas tout à fait en désuétude. Après maints débats durant les travaux de la Convention sur l'avenir de l'UE et l'échec du traité constitutionnel, le traité de Lisbonne consacre finalement une clause de solidarité qui s'en inspire ${ }^{4}$ : Au cas où un État-membre ferait l'objet d'une agression armée sur son territoire, les autres États-membres lui doivent aide et assistance par tous les moyens en leur pouvoir ${ }^{5}$; sans que cela n'affecte le caractère spécifique de la politique de sécurité et de défense de certains États-membres, ni la conformité avec les engagements souscrits au sein de l'OTAN, qui reste pour ses membres le fondement de leur défense collective et l'instance de sa mise en œuvre. Mais la PESD, si elle « inclut la définition progressive d'une politique de défense commune de l'Union [...], ne conduira à

\footnotetext{
${ }^{3}$ Créée en 1948, avant l'Alliance atlantique, dans un but de défense collective, mais rapidement marginalisée par celle-ci, l'UEO avait été pressentie au début des années 1990 pour canaliser les efforts des Européens au service du maintien de la paix et de la gestion de crises (voir ses «missions de Petersberg » définies en 1992 dans le château du même nom en Allemagne), parallèlement d'ailleurs aux nouvelles missions de l'OTAN. L'option d'une UEO «bras armé de l'UE» ('UEO ne compte pas en son sein tous les Etats membres de l'UE) sera délaissée en 1999 au profit de la construction d'une PESD au sein même de l'Union européenne.

${ }_{4}$ Article 42, alinéa 7.

${ }^{5}$ Conformément à l'article 51 de la Charte des Nations unies (concernant la légitime défense).
} 
une défense commune que lorsque le Conseil européen en aura décidé ainsi, à l'unanimité6 $»$.

La PESD, si elle fait partie intégrante de la politique étrangère et de sécurité commune de l'UE, la PESC, et représente pour celle-ci une capacité opérationnelle, ne se concentre pas sur la défense à proprement parler, mais bien sur la sécurité. Et plus particulièrement, sur les missions dites « de Petersberg », établies pour l’UEO en 1992.

Ces missions, ce sont elles qui fondent la Politique européenne de sécurité et de défense. Consacrées au sommet européen de Helsinki en 1999, confirmées par le traité de Nice un an plus tard, ces missions devaient être celles de la future «force de réaction rapide » de l'UE - cette « capacité militaire européenne autonome » de 60000 hommes établie comme l'objectif global de l'UE pour 2003 au plus tard : « des missions humanitaires et d'évacuation, de maintien de la paix, missions de forces de combat pour la gestion des crises, y compris des opérations de rétablissement de la paix ${ }^{7}$ ». Les origines et le consensus qui permettent à la PESD d'enfin exister sont là : cinq ans plus tôt, la guerre en Bosnie a confiné les européens dans une impuissance scandaleuse alors que la guerre sévit, trois années durant, aux portes de l'Italie, de l'Autriche et de la Grèce. La capacité militaire autonome qui va être mise en place est donc orientée tout entière vers ces missions de gestion des crises et de maintien de la paix qui doivent permettre à l'UE d'appuyer sa diplomatie et de rendre crédible ses objectifs lorsque c'est de la sécurité européenne qu'il s'agit.

Très rapidement, il est décidé de doter aussi la PESD de capacités civiles de gestion de crise ; en outre le consensus au Conseil admet la possibilité de déployer des missions hors d'Europe. En 2004, alors qu'un nouvel objectif global - pour 2010 cette fois - est discuté, deux nouveaux types de missions viennent élargir ces tâches de Petersberg initiales, à savoir l'appui au désarmement et à la lutte contre le terrorisme à la demande d'un État tiers ${ }^{8}$. Cependant, un texte fondamental, la Stratégie européenne de sécurité, rédigée sous la houlette de Javier Solana9 , fournit dès 2003 une portée plus large à la politique

\footnotetext{
${ }^{6}$ Article 42, alinéa 2.

7 Ancien article 17 du Traité sur l'Union européenne.

${ }^{8}$ L'article $43 \mathrm{du}$ Traité de Lisbonne (TUE) définit ainsi les missions de la PSDC (PESD) : ces missions « incluent les actions conjointes en matière de désarmement, les missions humanitaires et d'évacuation, les missions de conseil et d'assistance en matière militaire, les missions de prévention des conflits et de maintien de la paix, les missions de forces de combat pour la gestion des crises, y compris les missions de rétablissement de la paix et les opérations de stabilisation à la fin des conflits. Toutes ces missions peuvent contribuer à la lutte contre le terrorisme, y compris par le soutien apporté à des pays tiers pour combattre le terrorisme sur leur territoire ».

${ }_{9}^{9}$ Alors Haut Représentant pour la Politique étrangère et de sécurité commune (PESC) et Secrétaire général du Conseil (HRSG). Il occupera cette fonction pendant pas moins de dix ans.
} 
de gestion de crises et de maintien de la paix dont la PESD apparaît comme la pierre angulaire.

Cette stratégie de sécurité - une première pour l'UE - ne concerne pas seulement la gestion des crises et des conflits, ou la reconstruction. En s'intéressant au terrorisme international, à la criminalité organisée, la prolifération des armes de destruction massive, aux États faillis et aux causes socio-économiques des conflits, l’Union européenne développe une vision large de la sécurité internationale, au-delà de la sécurité territoriale enjeu prioritaire des politiques de défense traditionnelles, notamment pendant la guerre froide. Et c'est l'ensemble des moyens à disposition de l'Union qui doivent être mis en œuvre pour contrer ces menaces et favoriser la paix internationale. Pas seulement la PESD, mais l'ensemble des instruments et politiques qui forment son action extérieure.

Certains observateurs s'interrogent alors : l'Union aurait-elle vocation, à travers la PESD, à s'impliquer dans toutes les missions qui ne seraient pas des missions article $\mathrm{V}$, soit tout type d'opération militaire sauf celles relevant de la défense collective et donc de l'OTAN ? Entre cette assertion et la pratique, il y a toutefois un pas que nombre d'États-membres ne seraient pas prêts à franchir... en tout cas pas sous drapeau de l'UE. La question d'une Europe « able and willing ${ }^{10}$ » s'est posée à maintes reprises depuis l'insertion d'une dimension militaire au sein de la construction européenne. Et pour la plupart des États-membres, l'«Europe-puissance » est loin d'être au programme. Si nombre d'États se sont révélés « capables » et «volontaires » pour mettre leurs forces militaires au service de l'OTAN, de coalitions ad hoc ou de leurs alliés, la chose reste plus délicate dans le cadre de l'UE.

Ainsi, peut-on imaginer les troupes de l'UE prêtant main forte à l'ISAF'11 en Afghanistan? Ou déployées dans le cadre d'une opération de rétablissement de la paix manu militari? Difficile... mais pas impossible. L’UE a déjà mené des opérations militaires potentiellement coercitives, couvertes par un mandat sous Chapitre VII de la Charte des Nations unies ${ }^{12}$, bien que limitées dans le temps et dans l'espace. En outre, l'opération Atalante, lancée pour contrer le phénomène exponentiel de la piraterie maritime au large des côtes somaliennes et dans l'océan indien, ne relève ni du maintien de la paix ni de la défense collective contre un État (Frenay \& Glume, 2009). De manière

\footnotetext{
10 «Capable et volontaire », selon l'expression consacrée.

${ }^{11}$ Force internationale d'assistance à la sécurité en Afghanistan, commandée par l'OTAN depuis août 2003.

12 Intitulé « Mesures en cas de rupture de la paix, de menace contre la paix et la sécurité internationales, acte d'agression », le Chapitre VII implique en puissance la possibilité "d'utiliser tous les moyens nécessaire » pour rétablir la paix et la sécurité internationales, soit faire usage de la force armée, dans les conditions spécifiées par la résolution du Conseil de sécurité des Nations unies qui l'invoque.
} 
générale, depuis 2003, l’UE s'est toutefois cantonnée à des opérations PESD plutôt consensuelles - des missions de Petersberg au sens « originel».

Entre maintien de l'ordre dans les eaux internationales, gestion de crises en Afrique et maintien de la paix dans les Balkans, se pose la question de l'étendue exacte des opérations de l'UE. La "gestion des crises », le maintien de la paix et la prévention des conflits figurent en première ligne des objectifs de la PESD (Tardy 2009). Ce qui fait de l'UE, en principe, un acteur régional au service de la sécurité internationale entendue comme « bien commun » (Biscop 2005), c'est-à-dire notamment au service de la sécurité collective. Mais la pratique semble indiquer que lorsque les intérêts vitaux de l'Union européenne sont en jeu, les États-membres sont capable d'utiliser la PESD au service de leur intérêt commun - de façon, certes, encore limitée. C'est ainsi que pas à pas, l'Union européenne dépasse sans doute les tâches humanitaires et de maintien de la paix du début de la décennie pour rechercher une place, sur la scène internationale, en tant qu'acteur de sécurité à part entière.

\section{De l'utilité de l'intervention « civile »}

La force militaire n'est pas tout. La Stratégie européenne de sécurité, et sa remise à jour récente, le rappellent à grand renfort de rhétorique, et il ne pourrait en être autrement pour une Europe traditionnellement qualifiée de « géant économique » - nonobstant les difficultés récentes de la zone euro. D'autres instruments qui peuvent être mis au service de la politique étrangère, et en particulier de la gestion des crises, sont à disposition de l'UE depuis des années, voire des décennies. Ainsi en est-il des accords de coopération et de partenariat, de la politique commerciale commune, de ses clauses de conditionnalité politique, de la coopération au développement, mais aussi de la politique européenne de voisinage... autant de dimensions ${ }^{13}$ qui peuvent être rassemblées sous le concept de « politique étrangère structurelle » de l'Union européenne (Keukeleire 2001 : 536-551) si elles sont guidées par des objectifs communs, aujourd'hui énoncés dans le cadre de la PESC.

La Stratégie européenne de sécurité encourage aussi les efforts européens en matière de lutte anti-terroriste, les outils qui relèvent de la justice et des affaires intérieures, dans

\footnotetext{
${ }^{13}$ Les politiques évoquées ne sont pour la plupart pas conçues dès l'origine comme des instruments de politique étrangère. Cependant, à mesure que se développait la coopération politique européenne (la CPE, mécanisme ad hoc qui façonne dès les années 1970 une certaine politique extérieure de la Communauté européenne, sorte de prédécesseur de la PESC), on a assisté à une politisation certaine des relations économiques extérieures des Communautés européennes (De Wilde 1998). Et c'est l'ensemble des politiques de l'Union qui sont aujourd'hui plus ou moins empreintes de ses grandes orientations de politique étrangère.
} 
leurs aspects « externalisés ». Par ailleurs, phénomène plus récent, la Commission et le Conseil disposent d'instruments de gestion de crises et de prévention des conflits spécifiques, comme les observateurs électoraux, les représentants spéciaux, les sanctions économiques ou ciblées, l'aide humanitaire et la protection civile, l'Instrument de stabilité, ainsi que de fonds ou de budgets assortis... toute une palette d'instruments civils au sens de non militaires - au service «d'une Europe plus sûre dans un monde meilleur».

La PESD elle-même s'est très rapidement définie comme une politique de sécurité reposant sur des capacités à la fois militaires et civiles. Les États-membres de l'UE, s'inscrivant dans la tendance de l'ONU à diversifier les tâches et missions de ses opérations de paix, ont rapidement voulu doter la PESD de personnel civils au service de la sécurité : policiers, magistrats et juges, observateurs et conseillers politiques sont ainsi venus seconder les conseillers de la coopération « technique » des années 1990.

La première opération PESD de l'UE «à l'étranger » fut d'ailleurs une mission civile : la mission de police de l'Union européenne (MPUE) en Bosnie-Herzégovine prit le relais de celle des Nations unies en 2003, pour veiller au caractère démocratique des multiples forces de police de l'État fédéral complexe issu des accords de Dayton. Pour sa part, EUJUST Thémis en Géorgie avait pour but de seconder la réforme du système judiciaire après la «révolution des roses » de l'hiver 2003; tandis qu'après l'indépendance autoproclamée du Kosovo et le retrait de la MINUK après neuf ans d'administration du territoire, EULEX Kosovo relevait des deux aspects - judiciaire et policier. D'autres tâches - formation de forces de sécurité, appui au désarmement ou à un climat de sécurité, rétablissement de la confiance, soutien à l'État de droit - ont aussi été menées jusqu'ici par les personnels civils de la PESD. Et ce, aux quatre coins de la planète : en Afghanistan, en République démocratique du Congo (RDC), en Jordanie (pour l'Irak), en Indonésie (Aceh), dans les territoires palestiniens ou plus récemment en Guinée-Bissau.

Ces missions, moins télévisuelles qu'un déploiement de chars, restent peu connues du grand public. Elles constituent certainement des têtes-de-pont (plus ou moins conséquentes) pour les intérêts de l’Union européenne, qu'ils soient économiques, politiques ou sécuritaires. Cependant, comment jauger leur efficacité, voire même leur légitimité ? Certaines sont apparues comme des succès - la mission de désarmement à Aceh, où seule l'UE apparaissait impartiale - ou comme des opérations efficaces - la stabilisation pacifique de l'Ancienne république yougoslave de Macédoine (ARYM). D'autres sont dénoncées comme des tentatives inachevées - EUJUST Thémis en Géorgie - ou des pis-aller, comme la mission EULEX Kosovo, lancée alors que certains gouvernements de l'UE n'ont pas reconnu l'indépendance de l'ancienne province de la République fédérale de Yougoslavie. En termes de légitimité, il faut dire que la plupart de ces opérations sont menées avec l'accord ou à l'invitation des États tiers concernés; mais pour certains observateurs, ces missions «de conseil, de surveillance ou de formation» ne sont pas sans relents néo-colonialistes. 
À travers cette mosaïque de missions, c'est en tout cas le caractère multidimensionnel des instruments de l'Union européenne qui apparaît. Ces opérations civiles, ainsi que les opérations militaires de la PESD, n’interviennent pas dans un vacuum: Elles sont généralement encadrées par les autres politiques de l'Union, qui permettent d'appréhender une crise dans sa durée, de jouer sur la plupart de ses tenants et aboutissants. Ces politiques tantôt structurelles tantôt opérationnelles (Stewart 2006: 84-87) assurent à l'Union européenne une capacité d'action à court, moyen et long terme, face à une situation globale mais aussi lorsqu'une intervention ponctuelle s'avère nécessaire, tout spécialement dans le domaine de la gestion des crises et de la prévention des conflits. Le cas de la Bosnie-Herzégovine - et des Balkans dans leur ensemble - est édifiant: L'Union y a pris la relève de l'OTAN pour les opérations militaires de maintien de la paix ${ }^{14}$, y a progressivement déployé des missions civiles pour assister la reconstruction ou la transition, s'est liée aux États de la région par un Pacte de stabilité ${ }^{15}$ reposant notamment sur sa force économique, et ce sont tous les États des Balkans occidentaux - y compris la Serbie - qui sont soumis à l'effet d'entraînement d'un processus d'adhésion plus ou moins proche.

La PESD apparaît bien, à cet égard, comme la partie émergée de l'iceberg. Cependant, l'action multidimensionnelle a ses limites : celles de la cohérence, l'un des principaux défis pour l'action externe de l'UE. Que dire à cet égard des missions répétées menées ces dernières années en RDC, qui se soldèrent durant l'hiver 2008 par un refus de l'Union européenne de venir au secours de la MONUC à nouveau confrontée à la guerre au Kivu ? La fin de non-recevoir opposée alors à la demande du Secrétaire général de l'ONU apparut comme un puissant révélateur d'une politique « de non engagement » des Européens en RDC. Ou peut-être davantage, comme un révélateur de la divergence des intérêts des européens, qui certes développent consensuellement des stratégies communes, mais dont les intérêts vitaux restent affaire de priorités nationales. La facture des opérations militaires de la PESD ne relève-t-elle pas précisément de cette logique?

\section{Un catalogue des possibles}

Avant même l'acte officiel de naissance de la PESD, il était entendu que celle-ci s'érigerait selon une logique intergouvernementale. En conséquence, une certaine flexibilité s'avérait indispensable à son fonctionnement, à défaut de quoi elle n'aurait d'existence que théorique.

\footnotetext{
14 A l'exception du Kosovo, où la KFOR reste sous la houlette de l'OTAN, en attendant une EUKFOR

15 Voir le Pacte de stabilité pour l'Europe du Sud-Est.
} 
La logique intergouvernementale supposait des procédures de vote à l'unanimité au sein du Conseil. Cependant, la participation à la PESD et à ses opérations devant se faire sur base volontaire par les États-membres, l'unanimité devrait nécessairement être tempérée. Que se passerait-il si un État refusait de participer au processus ? L'opting out, soit la possibilité de «rester en dehors » de la PESD, a fourni une première réponse... au Danemark, seul État à l'avoir choisi jusqu'ici. Dans cette logique, il fallait aussi une certaine souplesse quant aux procédures de vote des opérations, auxquels tous les États-membres ne seraient pas tenus de participer. Il fut décidé qu'un État pouvait s'abstenir de voter une opération ayant des implications militaires sans «bloquer» les autres États-membres : l'abstention constructive ${ }^{16}$.

Il était aussi entendu que les forces militaires européennes seraient fournies « sur catalogue », c'est-à-dire qu'une série de capacités - hommes, matériels - seraient offerts par les États-membres pour constituer un catalogue (le catalogue de forces de Helsinki) dans lequel l'UE viendrait " puiser » pour construire sa capacité militaire autonome. Des forces potentielles listées par les États-membres, donc, mais en aucun cas une armée européenne : un «catalogue des possibles », en somme. En outre, une fois ce catalogue constitué - bien qu'il demeure constamment en mouvement, selon un processus de «génération de capacités » - il n'est pas dit que tous les États acceptent de donner le feu vert à leurs troupes pour une opération donnée ${ }^{17}$. Seuls les États-membres qui le souhaitent contribuent effectivement à telle ou telle opération. Et assument les coûts opérationnels ${ }^{18}$ et les risques militaires de celle-ci. Par contre, c'est l'ensemble des Étatsmembres qui, puisque l'opération est menée au nom de l'UE, en assument les risques politiques.

Dès lors, si tous les États-membres n'impliquent pas de troupes ou de capacités en soutien à l'opération militaire envisagée, un certain consensus doit exister en amont sur l'intérêt de l'opération, pour la sécurité européenne, voire pour la sécurité internationale - concept plus vague, parfois synonyme de fourre-tout, paravent d'intérêts particuliers - afin que les Vingt-Sept puissent prêter le drapeau européen à la mission. Mais si la France ou la Belgique ont intérêt à prêter main forte à l'ONU dans la région des Grands Lacs africains, comment convaincre les États baltes du bien-fondé d'une opéra-

16 D'autres mesures prévoient des procédures de vote à majorité qualifiée au Conseil des ministres, notamment lorsqu'une décision se prend sur base d'une stratégie ou d'une orientation précédemment adoptée par le Conseil, à l'unanimité.

${ }^{17}$ En outre, les forces du catalogue d'Helsinki ne sont pas « en attente » (stand-by), c'est-à-dire préidentifiées et «on call» durant un certain laps de temps, disponibles pour être déployées sur un théâtre donné le cas échéant. Seuls deux Battlegroups ou "groupement tactiques » le sont en permanence pour l'UE depuis 2007 (voir infra), ainsi que la Nato Response Force de l'OTAN.

18 Pour la part à charge du budget de l'UE et la clé de répartition entre Etats membres, voir «Athéna » et le mécanisme pour le financement des opérations PESD. 
tion militaire, même dédiée au maintien de la paix, dans des contextes où les troupes peuvent rapidement s'enliser, où la situation peut se dégrader avec des conséquences humanitaires et politiques potentiellement explosives? Il est parfois plus simple de ne pas prendre ce risque, et de laisser l'État-membre intéressé intervenir seul, en son nom propre - sans impliquer l’Union.

Néanmoins, lorsque plusieurs États-membres manifestent leur intérêt, la politique du Conseil a consisté ces dernières années à favoriser l'intervention de l'UE plutôt que l'intervention ad hoc. À défaut de quoi n'aurait été lancée aucune mission extraeuropéenne ; c'est-à-dire aucune opération militaire en dehors des Balkans, enjeu géopolitique au cœur de l'UE, dont la stabilisation était à la fois un objectif proprement européen, mais aussi une manière pour les atlantistes de démontrer leur bonne volonté à l'égard de Washington, qui souhaitait libérer l'OTAN de ses tâches de «baby-sitting » en Europe ${ }^{19}$. Ainsi, en quelque sorte, «l'européanisation » des dossiers liés aux grandes missions de la PESD - maintien de la paix et gestion des crises - existe sans aucun doute et est devenue une ligne directrice au sein du Conseil. À cet effet, deux traits apparaissent récurrents au fil des opérations : le rôle central d'une «nation leader» ou «nation cadre» (lead nation, frame nation) et les limitations imposées par le Conseil de l'UE au mandat de l'opération.

\section{Et sans les Français ?}

De toutes les opérations militaires lancées par l'Union européenne depuis que la PESD est véritablement opérationnelle ${ }^{20}$, soit depuis 2003, peu ont été réellement « européennes » - c'est-à-dire ont rassemblé la majorité des États-membres, à l'instar d'Althéa en Bosnie-Herzégovine. Cette opération, c'est un peu celle qui fonde la PESD, puisque c'est - entre autres évolutions - à la suite de la crise de l'Alliance atlantique (Haine 2004 : 251) sur la question d'une intervention militaire pour mettre fin à la guerre en Bosnie-Herzégovine que le consensus franco-britannique sur la PESD est

${ }^{19}$ Comme le titrait l'hebdomadaire The Economist dans un éditorial sur les nouvelles orientations de l'Alliance atlantique en mai 2003, quelques mois avant la reprise du commandement de la Force internationale d'assistance à la sécurité en Afghanistan (FIAS ou ISAF) par l'OTAN.

${ }^{20}$ L’UE est déclarée « opérationnelle » dès le Conseil européen de Laeken, fin 2001, parce qu'elle est à partir de ce moment dotée des organes nécessaires à la mise en œuvre des opérations extérieures relevant de la PESD : Conseil politique et de sécurité (COPS), Etat major de l'UE (EMUE) et Conseil militaire de l'UE (CMUE). Mais en réalité, ses forces militaires ne seront véritablement opérationnelles qu'après les arrangements dits de "Berlin Plus » (pour l'accès aux capacités et moyens de l'OTAN) qui interviendront fin 2002. En 2003 seront lancées à la fois la première opération civile (Mission de police de l'UE en Bosnie) et la première opération militaire (Concordia dans l'Ancienne République yougoslave de Macédoine) de la PESD. 
trouvé, trois ans plus tard, à Saint-Malo, en 1998. Ce consensus était la condition sine qua non pour lever le tabou propre à l'UE sur les «questions militaires et de sécurité »: Si le couple franco-allemand est traditionnellement vu comme le moteur de la construction européenne, en matière de défense - questions desquelles les Allemands demeurent à l'écart par la force de l'Histoire - la France et le Royaume-Uni sont les seules puissances capables de donner le tempo. Or, si Jacques Chirac et Tony Blair se mirent d'accord pour ouvrir la voie à la PESD, seule la France a cultivé depuis lors un rôle de leader en la matière - le Royaume-Uni privilégiant les engagements de l'OTAN.

Ce leadership s'est traduit, dans les opérations militaires de la PESD, par un volontarisme français sans lequel on compterait sans doute à ce jour... peu d'opérations du genre. En effet, seules les opérations en Bosnie et dans l'ARYM ont satisfait à la fois les partisans d'une défense européenne et les atlantistes. Pour les uns, il s'agissait de faire de la PESD une réalité ; pour les autres, d'assurer la relève des forces de l'OTAN appelées à d'autres desseins en Afghanistan... sans même évoquer l'Irak qui concentrait le gros des forces américaines en opérations. Dans la majorité des autres opérations militaires de l'Union européenne durant la décennie 2000-2010, la France a assumé le rôle de lead nation ou de frame nation - c'est-à-dire qu'elle a fourni la plupart des troupes et capacités, secondée de façon plus ou moins importante par d'autres contributeurs européens.

Ce fut d'abord le cas de l'opération Artémis dans la province congolaise de l'Ituri durant l'été 2003. Un cas certes un peu particulier : à la demande du Secrétaire général, la France a décidé de jouer les forces intérimaires pour donner le temps à la MONUC de se renforcer, devant la multiplication des exactions contre des civils et la résurgence des hostilités. Avant même le déploiement des soldats français, l'idée de placer cette force sous drapeau européen et d'ouvrir en conséquence la participation à d'autres Étatsmembres fut acceptée par le Conseil de l'UE. La France y conserva son rôle central, secondée par des Belges, des Suédois, des moyens britanniques notamment ; l'opération permit en outre de mettre à l'épreuve les nouvelles structures décisionnaires de la PESD (Neveux 2004).

Toujours en RDC, deux ans plus tard, l'opération EUFOR permit également de seconder la MONUC dans son effort de stabilisation durant les élections. La France y était à nouveau en bonne position. Enfin, en 2008, EUFOR Tchad-RCA est déployée pour sécuriser les zones frontalières peuplées de réfugiés du Darfour et préparer le terrain à la MINURCAT ${ }^{21}$. Encore une fois, EUFOR Tchad-RCA est largement française

\footnotetext{
${ }^{21}$ Mission des Nations unies en République centrafricaine et au Tchad.
} 
et voit le jour suite à l'engagement de la France dont les rapports avec le pouvoir tchadien ne sont méconnus pour personne ${ }^{22}$.

Mis à part les deux opérations de maintien de la paix ou de «sortie de crise » dans les Balkans, les autres engagements militaires au titre de la PESD ont donc été voulus, et assumés, principalement par la France et quelques autres États européens. Au point qu'on pourrait se demander: y aurait-il encore une PESD sans l'activisme des Français ? Y'aurait-il des opérations militaires en dehors des Balkans sans le confortable concept de «nation lead »? C'est l'enjeu de la cohésion des États-membres qui émerge ici comme une donnée cruciale pour l'avenir de la PESD - en tout cas en ce qui concerne son volet militaire.

Ceci nous ramène à la difficile question des intérêts et priorités des politiques étrangères des États-membres - et du dilemme potentiel avec un intérêt «proprement européen» (Frenay \& Glume 2009). Si «l'Europe» s'accorde à dire qu'il faut stabiliser l'Afrique, il est clair que la Belgique ou la France y sont davantage attentives que l'Autriche ou la Roumanie, pour lesquelles la priorité se déplace au contraire vers les Balkans ${ }^{23}$, ou les confins de la Russie et ses «conflits gelés »- désormais plus ou moins "dégelés », d'ailleurs ${ }^{24}$. Certes, tous les États européens se gaussent de l'importance de soutenir les Nations unies, notamment en Afrique, dans leurs efforts de maintien de la paix, au nom d'un «multilatéralisme efficace ${ }^{25}$ ». Toutefois, entre les déclarations de bonnes intentions et la décision d'engager ses propres troupes pour suppléer celles de l'ONU, le pas est rarement franchi.

En matière de maintien de la paix internationale, tout est question de priorités nationales - rarement, ou trop rarement, de priorités européennes communes. Ainsi, hormis les incontournables Balkans, pour lesquels le vide géopolitique aurait été insoutenable pour l'Europe, le bilan de la PESD ne semble-t-il pas révéler une faiblesse en termes de cohésion? Les opérations relevant d'un «intérêt vital» proprement européen ne se comptent-elles pas sur les doigts d'une main ? La PESD constitue-t-elle vraiment une capacité opérationnelle au service de la politique étrangère de l'Union, ou se confine-telle à un statut d'instrument marginal de prévention des conflits et de gestion des crises?

En dehors de l'Afrique, qui n'intéresse vraiment qu'une partie de l'Europe occidentale, les autres théâtres d'intervention d'intérêt « européen » sont soit laissés au fardeau

${ }^{22}$ La France a notamment des accords bilatéraux de défense avec le Tchad, ancienne colonie française.

${ }^{23}$ En 2010, l'Autriche prend le commandement de l'EUFOR en Bosnie, dont elle devient le plus gros contributeur... derrière la Turquie, en tant qu'Etat tiers associé à l'EUFOR.

${ }^{24}$ Nous faisons bien sûr référence à la guerre qui opposa la Russie et la province séparatiste géorgienne d'Ossétie du Sud à la Géorgie en août 2008.

${ }_{25}$ C'est l'un des concepts clés de la Stratégie européenne de sécurité. 
de l'OTAN (l'Afghanistan), soit occupés par des missions civiles, parce que l'intervention militaire est hors de portée - et ce, pas seulement pour les européens (Géorgie, Moldavie, territoires palestiniens, par exemple). Dans ce dernier cas, l'UE a d'ailleurs un train d'avance, car elle peut se servir de ses capacités civiles pour intervenir, nonobstant les réticences des États-membres eux-mêmes, ou des parties au conflit devant l'implication militaire d'un tiers (GLUME \& FRENAY, 2009, 23). Cependant, à un intérêt vital européen, c'est bien souvent l'intérêt de certains États européens qui se substitue: Vers l'Est pour les nouveaux États-membres, vers l'Asie centrale et le Moyen-Orient pour les Atlantistes, vers les anciennes colonies pour les États fondateurs. Le sens de la construction européenne ne serait-il pas précisément de réconcilier les intérêts des uns et des autres au nom d'un intérêt supérieur de l'Union?

\section{Mandats limités mais réalistes}

Manque de vision ou prudence politique, cette réticence à s'engager militairement et à s'engager ensemble n'est pas étrangère aux décisions qu'a prises le Conseil quant aux mandats qu'il a fallu définir pour ces opérations militaires menées sous le sceau de la flexibilité. Chaque fois, le mandat a été soigneusement limité, de façon à ce que tous les États-membres de l'UE, à défaut d'être contributeurs sur le terrain, puissent assumer les risques politiques d'un engagement militaire sous la bannière étoilée : limitation de la mission dans l'espace, dans le temps, en termes de tâches mais aussi d'objectifs.

Artémis fut établie pour un été, le temps pour les Français d'empêcher l'extension des massacres et la contamination à toute la région, voire toute la RDC, du conflit en Ituri. Un été ; le temps pour la MONUC devenue incapable de protéger les civils de rassembler davantage de troupes et de capacités militaires. Et cette mission fut limitée à la ville de Bunia, chef lieu de l'Ituri, et à ses environs : pour permettre aux déplacés de revenir dans cette zone « sécurisée ", pour éviter aussi que les violences n'embourbent les soldats de l'Europe sur un théâtre trop vaste pour être contrôlé. Idem pour l'EUFOR en RDC : sécuriser Kinshasa, centre symbolique du pouvoir, durant la période des élections a évité un engagement trop important des Européens dans un pays loin d'être totalement pacifié. Peut-être, au fil du temps, ces limites se distendent-elles : EUFOR Tchad-RCA s'étala sur une année entière, dans deux États, avec une tâche principale de stabilisation, toujours en soutien à l'ONU. Quant au soutien de l'UE à la Mission de l'Union africaine au Soudan (MUAS), il dura plusieurs années mais n'impliqua pas les militaires sur le théâtre du Darfour : il fut principalement financier, logistique et opérationnel.

Ce que les européens (et de manière générale, les militaires) veulent éviter à tout prix, c'est bien sûr que le contrôle d'une situation leur échappe - et que les troupes engagées en leur nom se trouvent embourbées dans une guerre qui n'est pas la leur. Pour les pays non contributeurs, la limitation du mandat doit éviter que leurs gouvernements se voient interpeler en cas d'extension des hostilités : pourquoi des opérations militaires 
qui ne relèvent pas de notre intérêt vital, à travers leur sceau européen, impliquent-elles notre réputation, notre pays, notre peuple?

Un mandat limité, c'est aussi la garantie de troupes vite déployées et vite rapatriées, soit de missions courtes : en effet, le risque majeur des opérations de maintien de la paix aujourd'hui, ce n'est pas forcément le bourbier. C'est aussi celui d'un déploiement sans fin, parce que désormais, « l'état final recherché » assigné aux militaires, c'est-à-dire l'objectif politique de l'opération de paix traduit en missions militaires, est bien souvent hors d'atteinte. Parce que se multiplient d'une part les situations de ni-guerre ni-paix comme par exemple au Sud Liban -, des situations fragiles où les hostilités ont cessé mais où un accord de paix est hors de vue, et d'autre part les États faillis ou quasi faillis - comme en Somalie ou en RDC - où déployer des troupes signifie suppléer un gouvernement national ad vitam aeternam. Et l'UE ne peut souffrir une mobilisation à long terme de ses troupes, sous peine de voir immobilisée sa capacité militaire autonome. Avec deux millions de personnels sous les drapeaux, on s'accorde à dire que les États européens ne sont capables de déployer que 100000 soldats en opérations extérieures (Keohane 2008 : 26). Ce chiffre est encore bien moindre si l'on considère la capacité de projection de forces que les États-membres allouent à l'Union européenne.

Une autre donne contribue enfin à expliquer ces limites : ces opérations ont été mises en œuvre en renfort des Nations unies, pour prêter main forte à des casques bleus qui n'ont pas les moyens d'assumer un changement de mandat du jour au lendemain, ou le lancement rapide d'une opération ; mais qui demeurent la principale force de paix impliquée au nom d'un intérêt, cette fois, véritablement collectif. Ces casques bleus sont précisément ceux qui sont désormais chargés de ces mandats formidables - pacifier un pays entier, en protéger les civils, assurer désarmement, maintien de l'ordre et sécurité, surveiller des accords de paix remis en cause de toutes parts - voire de «missions impossibles ».

\section{Entre rôle d'appui et d'appoint}

Si de nombreuses opérations européennes ont eu lieu en appui à l'ONU, c'est parce que l'UE en a fait l'une des priorités de la PESD. En 2005 et 2007, deux documentscadre coordonnent la PESD avec les Nations unies. Et dans le cadre des fonctions de gestion de crises et maintien de la paix adoptées à Helsinki, deux concepts d'opération vont émerger: la Bridging operation et la Stand-by operation (Liegeois 2006 : 151). Soit, d'une part, les opérations devant assurer «l'interim» pendant le renforcement ou l'évolution d'une opération de maintien de la paix onusienne ; d'autre part, une opération de réaction rapide préparant le terrain à une opération onusienne - dont tout le 
monde sait que leur mise en place est un chemin tortueux, en tout cas trop lent pour qu'un conflit ne les attende ${ }^{26}$.

Que les européens veuillent renforcer par la PESD la capacité d'action des casques bleus n'est qu'un juste retour des choses, si l'on se rappelle que la majorité des Étatsmembres de l'UE ne fournissent plus, ou peu, de casques bleus à l'ONU. Ces derniers proviennent désormais essentiellement de pays en développement ou de puissances émergentes ${ }^{27}$. S'impliquer dans les missions de maintien de la paix via leurs propres institutions régionales est un choix que les européens ont fait au milieu des années 1990, après les graves difficultés de ceux-ci en Bosnie et au Rwanda notamment - qui donnèrent lieu à une crise de l'ONU, mais surtout débouchèrent sur les derniers génocides du XXe siècle. En intervenant pour soutenir l'ONU, l'Union européenne s'adapte ainsi à une pratique nouvelle du maintien de la paix que ses États-membres ont contribué à asseoir... en évitant d'immobiliser des troupes dans des missions au long cours : Au vu des mandats des opérations de l'UE, on pourrait même parler d'opérations «touch and $g 0^{28} »$.

Ces coopérations mises en place par l'Union européenne ne concernent pas seulement l'ONU. Elles ont été également développées avec l'OTAN, qui fut d'ailleurs la première organisation régionale à se déclarer "au service de l'ONU et de la $\operatorname{CSCE}^{29}$ » dès 1992, pour des missions de gestion de crises et de maintien de la paix... au cas par cas et sous réserve d'un accord du Conseil de l'atlantique nord. Dans un contexte de fin de guerre froide favorable à des mutations géopolitiques parfois violentes, l'Organisation du traité de l'atlantique nord avait, de façon précoce, voulu pérenniser une existence que la seule défense collective ne légitimait plus.

Dans le cadre de la transformation de l'Alliance et de ses missions, des discussions furent engagées dès 1994 avec l'UE pour favoriser le développement d'un " pilier européen » dans l'OTAN - notamment à travers une coopération de cette dernière avec l'Union de l'Europe occidentale. Les missions de Petersberg alors endossées par celle-ci

\footnotetext{
${ }^{26}$ Le «système de forces en attente » des Nations unies, par lequel ses États-membres mettent à disposition des opérations de maintien de la paix des listes de personnels, demeure soumis à la décision finale des autorités nationales lorsque le Secrétaire général requiert des capacités militaires pour une opération donnée. Il est dès lors insuffisamment fiable pour assurer la génération rapide de forces - alors qu'une des conditions principales de réussite de ces opérations est précisément la rapidité de l'intervention, avant que ne se démultiplie la violence.

${ }^{27}$ Les contingents provenant de pays occidentaux représentaient $40 \%$ des casques bleus à la fin des années 1990, mais tombent sous la barre des $10 \%$ à partir du début de la décennie 2000 (Guehenno 2005 : 26).

${ }^{28}$ L'expression est de Fabien Limonier, «Europe in the Balkans after 2004 », communication lors de l'International workshop on European Peace Building, CECRI-UCL \& Nelson Mandela Centre for Peace and Conflict Resolution, Bruxelles, 25 février 2010.

${ }^{29}$ Avant qu'elle ne devienne l'OSCE en 1995.
} 
allaient donner lieu à de premières expériences conjointes dans les Balkans - surveillance de la zone d'exclusion aérienne en Bosnie et de l'embargo en Adriatique - et favoriser l'idée d'un accès des européens aux capacités de l'OTAN. Ces discussions, dites «de Berlin » du nom de la capitale hôte du sommet atlantique qui annonça leur lancement, débouchèrent fin 2002 sur les arrangements de «Berlin Plus » donnant à l'UE un accès aux moyens et capacités de l'OTAN.

Ces arrangements impliquent un accès aux équipements de l'Alliance, mais aussi à ses capacités de planification - prodiguant un temps à Washington un contrôle « résiduel » sur la PESD. En outre, une des conditions posées par les Américains au développement de la PESD fut le refus des $3 \mathrm{D}$ : pas de duplication, pas de découplage ni de discrimination ${ }^{30}$. En somme, il fut alors décidé que l'UE n'interviendrait pas militairement là où l'OTAN serait déjà engagée - cette dernière étant prioritaire, le cas échéant, sur l'allocation des troupes notamment ${ }^{31}$.

Néanmoins, les coopérations les plus innovantes sont sans doute celles qui ont été développées, pas à pas depuis 2005, avec l'Union africaine, autre grande organisation s'étant déclarée au service de la sécurité collective régionale (Liegeois 2010). Sur une demande de sa présidence tchadienne, au printemps 2005, soit après les épisodes les plus tragiques d'un conflit déjà bien avancé au Darfour, l'UE avait accepté de soutenir la Mission de l'UA au Soudan. Cette dernière était alors la seule force multinationale acceptée par Khartoum - une force dont le mandat, et dès lors les effectifs nécessaires, s'élargiront successivement, de l'observation du cessez-le-feu à la protection des civils dans la région en guerre. Le rôle des européens va d'abord être financier : la MUAS sera largement financée par la Commission européenne, à travers la création de l'African Peace Facility. Les Européens assumeront également une opération civilo-militaire pour soutenir la $\mathrm{MUAS}^{32}$.

Mais l'essentiel est ailleurs. L'essentiel, c'est le partenariat développé par l'UE avec l'Union africaine, un partenariat qui dépasse le cas du Darfour. Les demandes de Kofi Annan au printemps 2005, à la veille d'une énième réforme de l'ONU, allaient en ce

\footnotetext{
${ }^{30}$ Pas de duplication inutile des équipements, ni de découplage des alliés, ni de discrimination entre les membres de l'OTAN non membres de l'UE (comme la Turquie).

31 Il n'y a ni «armée » de l'OTAN ni «armée » de l'UE. Les militaires restent ceux des Etats membres dans les deux cas. Pour les opérations extérieures, en cas de demande d'une de ces organisations, chaque Etat membre décide de sa participation. Ainsi, aujourd'hui, le même soldat belge envoyé en 2008 en mission pour l'OTAN en Afghanistan, peut avoir été déployé sous bannière de l'UE au Tchad en 2009.

32 Fourniture d'équipements, de véhicules, appui à la planification du pan civil de la mission, aide à la rotation aérienne des troupes notamment. Ainsi l'UE assurera la rotation de plus de 2000 soldats de l'UA au Darfour - contre plus de 28.000 pour l'OTAN, qui répondra aussi positivement à la demande de l'Organisation panafricaine.
} 
sens. Partant du constat que les opérations de maintien de la paix n'étaient plus seulement le fait de l'ONU, mais depuis plus d'une décennie, une tâche également endossée par certaines organisations régionales, le Secrétaire général exhortait les États-membres à soutenir l'ONU, mais aussi le développement des capacités africaines de maintien de la paix. Progressivement, l'UE a commencé à répondre à ce souhait. D'autres organisations multilatérales, ou accords bilatéraux, avaient déjà lancé pareilles initiatives, allant jusqu'à inscrire leur financement au titre de la coopération au développement ${ }^{33}$. En la matière, l'Union européenne pouvait être un bailleur de fonds de premier ordre. Il eut été inconcevable de ne pas soutenir les efforts d'une organisation panafricaine aspirant à la sécurité collective régionale.

A travers de ces initiatives et mécanismes de coopération, l’Union européenne a développé un rôle spécifique au sein du système de sécurité collective global de l'ONU. Un rôle de garant de la sécurité collective régionale - en Europe - et un rôle d'appui au Système des Nations unies et à ses partenaires dans le maintien de la paix, au-delà des frontières européennes.

\section{Des groupements tactiques en (longue) attente}

Les Battlegroups européens semblent renchérir à la fois sur cette logique de coopération et sur ce rôle d'appui au Système onusien. La capacité militaire autonome de l'UE, qui devait être mise en place en 2003 d'après l'Objectif global de Helsinki, devait s'avérer insuffisamment adaptée à la PESD telle que les décideurs européens commençaient à la concevoir : pas assez flexible, pas assez rapide. Sans parler de la difficulté, pour les États-membres, d'assurer la disponibilité de 60000 hommes capables d'être déployés une année durant sur un théâtre ${ }^{34}$. Concept issu des guerres balkaniques, l'Objectif global 2003 apparaissait trop peu flexible pour des opérations hors d'Europe, alors même que la stabilisation dans les Balkans n'allait manifestement requérir qu'une dizaine de milliers d'hommes pour relever l'OTAN, exception faite du Kosovo. Réponse pragmatique, le concept de Battlegroup (BG), ou "groupement tactique interarmées », fut donc mis en œuvre dès 2004. D’inspiration allemande, le concept est censé, depuis 2007, constituer l'un des piliers opérationnels de la politique de gestion militaire des crises de l'UE.

Aujourd'hui, l'UE peut compter en permanence sur deux groupements tactiques en attente. Ces Battlegroups sont des forces militaires multinationales de la taille d'un bataillon, soit 1500 hommes, armés et comme leur nom l'indique, préparés au combat. Ren-

\footnotetext{
${ }^{33}$ Le programme français RECAMP, par exemple.

${ }^{34}$ Avec les rotations des soldats tous les 4 mois environ, comptez 180000 militaires par an.
} 
forcés par des éléments d'appui et de soutien logistique, ils sont entraînés pour être déployés rapidement afin d'assumer, à la demande du Conseil de l'UE, tout le spectre des missions de Petersberg. Ainsi, un BG peut en principe être déployé dans un délai de cinq à dix jours pour des missions d'une durée initiale de 30 jours, pouvant aller jusqu'à quatre mois (120 jours). La majorité des États-membres ${ }^{35}$ se sont engagés, dès 2004, à mettre sur pied pas moins de seize BG. Depuis janvier 2007, l’UE dispose en permanence de deux BG opérationnels en attente, selon une rotation préétablie qui intervient tous les six mois (soit quatre par an).

Ces unités militaires flexibles, multinationales et bien entraînées pourraient intervenir pour arrêter l'expansion d'un conflit localisé, sécuriser l'apport d'aide humanitaire, soutenir des Casques bleus en difficulté ou protéger des civils victimes d'atrocités massives, notamment dans le cadre du principe de la "responsabilité de protéger» adopté par l'Assemblée générale des Nations unies fin 2005 (Glume \& Martens 2009). Si l'on s'en tient aux leçons des années 1990 et aux nécessités de la protection des populations civiles, devenue une préoccupation récurrente du Conseil de sécurité de l'ONU, le concept semble effectivement déborder de potentiel. Cependant, trois ans après qu'ils aient été déclarés opérationnels et mis à sa disposition, l'Union européenne n'a pas (encore) eu recours aux Battlegroups. Faute d'opportunité... ou de consensus.

Tandis que le paysage des opérations de paix semble gagner en cohérence - après les évolutions substantielles postérieures à la fin de la guerre froide - l'Union européenne paraît avoir défini son rôle, certes limité et spécifique, au sein du Système onusien, le seul vraiment universel, qui tente d'assurer la paix et la sécurité internationales. Un rôle qui, en se donnant pour idéal la paix et la sécurité internationale comme horizon, manque cependant de cohésion dans sa mise en œuvre. Et pour cause : la paix et la sécurité internationales ne se traduisent en intérêt vitaux que lorsque la sécurité du continent est directement menacée - ou perçue comme telle par les Vingt-Sept.

\section{Conclusion : À la recherche d'une politique étran- gère commune}

A travers les politiques qui encadrent la gestion des crises et la prévention des conflits, à travers les opérations civiles et militaires de la PESD et les mécanismes de coopération pour le maintien de la paix, les États-membres de l’Union européenne lui ont défini un rôle spécifique en matière de sécurité. Un rôle principalement axé sur la prévention et la gestion des crises et des conflits, ainsi que sur la reconstruction d'États ravagés par la guerre. Un rôle qui échappe désormais partiellement aux États-membres,

35 A l'exception du Danemark et de Chypre notamment. 
qui ont fait de l'Union un acteur international à part entière. C'est en effet l'ensemble des institutions et politiques de l'Union - du Conseil à la Commission, du Dialogue politique à la coopération au développement - qui sont désormais sollicités par la Stratégie européenne de sécurité, dont la Politique européenne de sécurité et de défense n'est que le fer de lance opérationnel. L'Union européenne, au fil du développement de la PESD et de ses instruments, s'est également définie une place au sein d'un système de sécurité collective, celui de l'ONU, qui a sans doute connu plus de mutations ces vingt dernières années que depuis sa création, en 1945 à San Francisco.

Cependant, ce rôle et cette place connaissent aujourd'hui des limites encore prégnantes; des limites qui ont sans doute à voir avec la jeunesse de la PESD, mais qui pourraient devenir des tendances lourdes - voire un fardeau - pour l'action externe de l'Union si ses États-membres ne s'engagent pas à les dépasser.

En dépit de l'action structurelle constante que l'Union européenne tend à exercer sur son environnement, en dépit des instruments opérationnels dont elle dispose aujourd'hui, elle demeure marquée par un dilemme crucial : voulant apparaittre comme un acteur bienveillant au service de la sécurité internationale conçue comme un bien commun, conformément à la tradition d'une construction européenne rejetant les logiques de puissance brutes, l'UE demeure soumise à la prégnance de ses intérêts vitaux. L'image «altruiste » qu'elle s'est construite à force de consensus ne peut éluder la nécessité d'un consensus plus profond, à défaut de quoi elle risque de virer à la peau de chagrin. En effet, si l'on peut affirmer que la PESD constitue bel et bien un instrument de choix au service de la gestion de crise et du maintien de la paix, il est plus difficile de voir en elle l'instrument d'une politique étrangère européenne à part entière.

Certes, à travers ses opérations et ses politiques pour les Balkans ou dans le Caucase, dans la Géorgie en proie à la guerre, ou dans l'Océan indien où ses intérêts commerciaux sont menacés, l'Union européenne a montré qu'elle avait la volonté et les moyens d'avoir un impact décisif sur sa propre sécurité, sur la sécurité européenne. En Europe, ou dans la "Grande Europe », celle de l'Union élargie, celle du voisinage de la Russie, voire du Moyen-Orient, les Vingt-Sept ont pu trouver la cohésion nécessaire à l'action commune. Cette cohésion peut être analysée comme la manifestation de l'existence d'un intérêt proprement européen, d'un intérêt vital de l'UE, ou d'une cohérence des objectifs de politique étrangère et de sécurité des États-membres.

Toutefois, cette cohérence est l'apanage des enjeux qui menacent l'Union européenne à ses portes. En matière de gestion de crises et de maintien de la paix, le consensus se fait plus évanescent une fois ces frontières dépassées. Au-delà, les intérêts manifestés par certains États-membres peuvent certes avoir un effet d'entraînement sur leurs pairs, mais uniquement au profit de l'ONU et de son multilatéralisme, défini par l'UE comme une priorité, ou la pierre angulaire d'une sécurité globale. L'ensemble de ces interventions - civiles et militaires - et des politiques qui les appuient, définissent la position, le rôle de l'UE au sein du système de sécurité collective international. Un rôle « d'appoint» en soutien à l'ONU, même quand les intérêts vitaux de l'Europe ne sont 
pas remis en cause. Mais hormis ces incursions ponctuelles au service de la sécurité internationale, au nom du consensus affiché sur l'importance du Système des Nations unies, ce sont toujours les politiques étrangères nationales qui prévalent. En somme, une politique de gestion des conflits ne remplace pas une politique étrangère, a fortiori quand celles-ci doivent être communes. Sans la seconde, la première est vouée à s'essouffler.

En aucun cas l'absence d'opérations de la PESD dans certaines régions du monde ne peut inférer l'absence d'une politique étrangère de l'UE, qui dispose d'autres moyens collectifs d'envergure pour son action externe. Toutefois, le manque de cohésion constaté une fois que les crises ou les opérations se font plus lointaines, le manque de propension à s'impliquer et à prendre des risques collectivement, suggère que la PESD n'est pas ou pas encore conçue par les européens comme un instrument au service de leur politique étrangère, la PESC. Ou bien la PESC n'est pas encore assez tangible pour les capitales, ou bien elle se repose davantage sur les politiques structurelles de l'Union plutôt que sur ses outils opérationnels, incarnés par la PESD, une fois débordées les frontières de la Grande Europe. C'est le cas pour l'Afghanistan ou l'Afrique, où les intérêts vitaux de l'UE sont plus difficiles à définir à Vingt-Sept que dans les Balkans occidentaux, aux portes du Caucase ou au large de la corne de l'Afrique. Dans ces derniers cas, la PESD a été utilisée de manière réactive, pour soutenir la diplomatie de l'Union, de façon à contribuer à protéger les intérêts directs de l'Union européenne - des intérêts éminemment politiques, liés à la stabilité du continent, mais aussi à la sécurité énergétique et commerciale des Vingt-Sept. Au-delà, la PESD apparait trop rarement comme un instrument opérationnel pouvant soutenir les positions diplomatiques de l'Union sa politique étrangère.

Dix ans de PESD ont fait de l'Union européenne un acteur visible de la sécurité internationale, et il faut le répéter, la PESD n'est que la partie émergée de l'iceberg. Mais cet acteur ne semble manifester sa cohésion que dans la gestion des crises et des conflits sur les marches de l'Empire. La position que l'institution occupe, au sein du système de sécurité collective incarné par l'ONU, relève de cette logique : elle constitue pour le secrétaire général une garantie de sécurité collective régionale, en Europe, ainsi qu'un instrument potentiel au service des opérations de paix. Mais un instrument dont la disponibilité est marquée par l'incertitude, et la part du fardeau bien ténue, une fois remisés les intérêts de sécurité «vitaux » de l’Union.

Ces intérêts vitaux de l'Europe, qui font l'objet de l'une ou l'autre «stratégie commune » régionale du Conseil, ne sont plus aussi tangibles lorsque c'est de politique mondiale qu'il s'agit. La toute-consensuelle Stratégie de sécurité européenne dénote une approche commune certaine, mais la sécurité internationale comme «bien commun» n’est pas un moteur de cohésion suffisant au niveau opérationnel. En conséquence, l'Union européenne devra résoudre le hiatus entre son image de «puissance civile bienveillante » et ses intérêts - osons le mot - stratégiques.

En dix ans, la PESD s'est développée de manière plus importante que les eurosceptiques n'auraient pu l'imaginer, nonobstant les dernières crises de la construction euro- 
péenne. Elle a dépassé le «stade Petersberg» et continue à être dotée de nouveaux instruments opérationnels, reposant sur des politiques communes dont les objectifs dépassent la prévention de la violence, de son expansion, de sa récurrence - utilisant le meilleur que l'UE avait à offrir. Mais elle doit pouvoir être guidée par une véritable politique étrangère commune. À défaut de quoi, la fin espérée des conflits en Europe et le manque de cohésion sur les enjeux extra-européens viendront à bout d'une politique de gestion des conflits euro-centriste. Le traité de Lisbonne a donné à la PESD un nouveau nom : la politique européenne de sécurité et de défense est devenue, à l'orée de l'année 2010, la PSDC, la politique de sécurité et de défense commune. Reste désormais aux européens à traduire le sens de leurs intérêts particuliers en intérêts communs.

\section{Bibliographie sélective}

BISCOP Sven (2005), The European Security Strategy : A Global Agenda for Positive Power, Aldershot/Burlington, Ashgate.

CHETAIL Vincent (ed.) (2009), Post-Conflict Peacebuilding: A Lexicon, Oxford/New York, Oxford University Press.

DE WILDE D’ESTMAEL Tanguy (1998), La dimension politique des relations économiques extérieures de la Communauté européenne. Sanctions et incitants économiques comme moyens de politique étrangère, Bruxelles, Bruylant.

DURCH William J. \& BERKMAN Tobias C. (2006), «Définitions et délimitation des opérations de paix », in COULON, Jocelyn, Guide du maintien de la paix 2007, Outremont (Québec), Athéna-CEPES.

FRENAY Marie \& GLUME Galia (2009), «Du Caucase aux côtes somaliennes : l'Union en opérations extérieures", in Euros du Village.eu, mis en ligne le 31 décembre 2009.

GLUME Galia (2008) : «Nouveaux théâtres, nouvelles missions? Les opérations de paix au sein des populations », in STRUYE DE SWIELANDE, Tanguy (dir.), Les interventions militaires en zones urbaines: paradigmes, stratégies et enjeux, Bruxelles, Bruylant, pp.117-140.

GLUME Galia \& FRENAY Marie (2009), «Un an après la guerre avec la Russie. La Géorgie: cas d'école d'une pensée stratégique en évolution», in Défense et sécurité internationale, $\mathrm{n}^{\circ} 52$, octobre 2009.

GLUME Galia \& MARTENS Quentin (2009), «La Responsabilité de protéger : où en est l'Union européenne? ", in Les Cahiers du RMES, vol.VI, n¹, été/automne 2009. 
GUEHENNO Jean-Marie (2005), "Rapport Brahimi, cinq ans après », in COULON, Jocelyn (dir.), Guide du Maintien de la paix 2006, Québec (Outremont), éd. Athéna/CEPES, pp.20-33.

HAINE Jean-Yves (2004), Les États-Unis ont-ils besoin d'alliés?, Paris, Payot.

KEUKELEIRE Stefan (2001), «Au-delà de la PESC : la politique étrangère structurelle de l'Union européenne », in Annuaire français des Relations internationales, vol.2, Paris, La Documentation française, pp.537-551.

KEOHANE Daniel (2008), "European military capabilities. Sharing the burden », in European security and Defence Policy Review, Issue 6, July 2008.

LIEGEOIS Michel (2006), «L’Union européenne et les opérations de paix : l'arrivée à maturité d'un nouvel acteur? ", in COULON, Jocelyn (dir.), Guide du maintien de la paix 2007, Outremont (Québec), Athéna/CEPES, pp.143-160.

LIEGEOIS Michel (2010), « Le rôle des organisations européennes dans le maintien de la paix et de la sécurité internationale » in PELLON, Gaëlle \& LIEGEOIS, Michel (dir.), La gestion des conflits dans la Grande Europe, Bruxelles, P.I.E.-Peter Lang/CECRI, collection Géopolitique et résolution des conflits, sous presse.

MOORE Rebecca R. (2007), NATO's New Missions. Projecting Stability in a Post-Cold War World, Westport/London, Praeger Security International.

NEVEUX Bruno (2004), "Vers une Union opérationnelle? Artémis », in Défense nationale, $\mathrm{n}^{\circ} 5$.

STEWART Emma (2006), European Union Conflict Prevention: Policy Evolution and Outcome, Berlin, Kieler Schriften zur Friedenswissenschaft, LIT Verlag.

TARDY Thierry (2009), Gestion de crise, maintien et consolidation de la paix. Acteurs, activités, défis, Bruxelles, De Boeck, collection Crisis. 